\title{
Gastrointestinal bleeding with joint pain
}

\author{
A.K. Singh, C.L. Birda, J. Samanta, S.K. Sinha, R. Kochhar \\ Department of Gastroenterology, Postgraduate Institute of Medical Education and Research (PGIMER), Chandigarh-160012, India.
}

\section{Quiz}

A 22-year-old male presented with fever, diffuse abdominal pain and melena for 2 days. He also had symmetric polyarthralgia involving knee and ankle. Physical examination showed no peripheral lymphadenopathy or joint swellings. Investigations showed normal blood count, kidney function tests with raised serum transaminases, AST $76 \mathrm{U} / \mathrm{L}$ and ALT $82 \mathrm{U} / \mathrm{L}$ (normal $<40 \mathrm{U} / \mathrm{L}$ ). Urinalysis revealed no hematuria or proteinuria. Computed tomography (CT) abdomen showed diffuse thickening of small bowel (Figure 1A). Esophagogastroduodenoscopy showed hyperemic edematous mucosa with ulcerations in the duodenum (Figure 1B). After two days patient developed multiple reddish purpura over bilateral lower limbs (Figure 2). What is the possible diagnosis?

\section{Answer}

Based on symptoms of fever, joint pain, gastrointestinal symptoms and palpable purpuras in this patient, a clinical diagnosis of IgA vasculitis with gastrointestinal (GI) involvement was made in accordance with European League against Rheumatism (EuLAR) and Pediatric Rheumatology Society (PReS) 2006 criteria. Thickened small bowel on CT can be seen in various conditions including bowel ischemia, hypoproteinemia, Crohn's disease or vasculitis. Duodenal histopathology showed evidence of small vessel vasculitis, confirming the diagnosis. Patient was given acetaminophen and oral prednisolone $(1 \mathrm{mg} / \mathrm{kg} / \mathrm{d})$ and became afebrile, pain free and lesions start decreasing after 7 days. Prednisolone was gradually tapered over 4 weeks. He is doing well at a follow up of 2 years.

IgA vasculitis, previously known as Henoch-Schonlein purpura (HSP), is a small vessel vasculitis mediated by Type III hypersensitivity with IgA deposits in the vessel wall. It is a multisystem disease characterised by tetrad of palpable purpura, arthritis, glomerulonephritis and gastrointestinal manifestations. Though common in childhood, young adults are known to be affected (1). Palpable skin lesions are the earliest manifestations in approximately $70 \%$ of the patients, typically affecting lower limbs and gluteal region.

GI involvement occurs in 50-75\% patients and presents with colicky abdominal pain, vomiting or gastrointestinal bleeding. In 10-15\% patients, gastrointestinal involvement

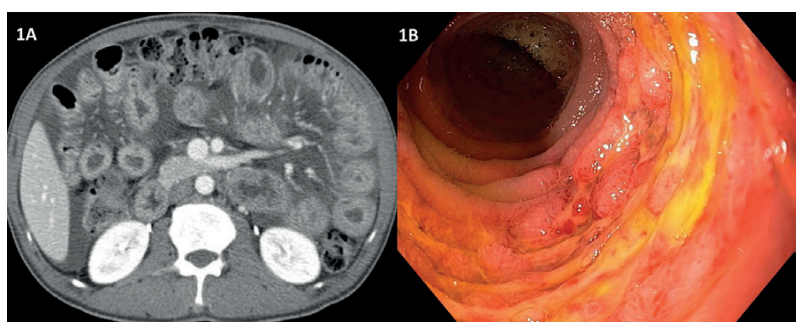

Figure 1. - Computed tomography (CT) abdomen (fig. 1a) showing diffuse thickened small bowel loops. Endoscopic image from duodenum (fig. 1b) showing hyperemic edematous mucosa with ulcerations.

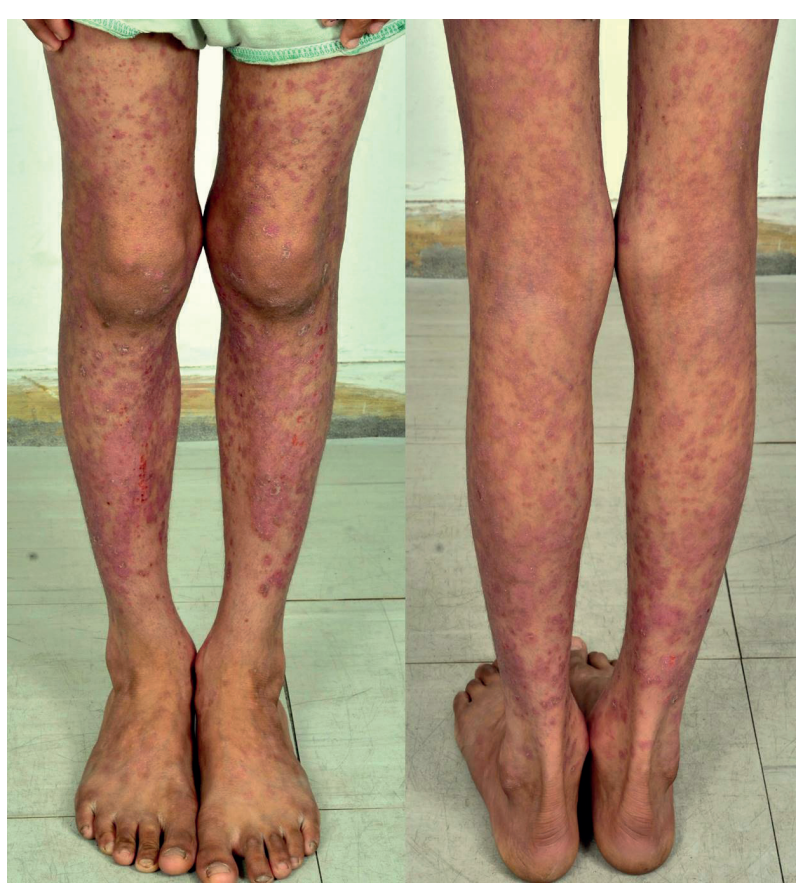

Figure 2. - Clinical picture showing multiple palpable purpuras over the lower limbs involving both extensor and flexor aspects.

precedes the appearance of skin lesions, making diagnosis of IgA vasculitis difficult to establish as in the present case (3). IgA vasculitis resolves spontaneously in most of the cases and uncomplicated cases are managed with

Correspondence to : Professor Rakesh Kochhar, Department of Gastroenterology, Post-Graduate institute of medical education and research (PGIMER), Chandigarh, India- 160012. Phone : +911722756608

Email :dr_kochhar@hotmail.com

Submission date : 18/04/2020

Acceptance date : 18/05/2020 
supportive care. Severe GI symptoms and severe rash improves rapidly with oral steroids.

\section{Conflict of interest}

All the authors have no potential conflicts of interest to disclose with respect to research, authorship and publication of this article.

\section{Funding}

The authors received no financial support for their research, authorship and publication of this article.

\section{Consent}

Patient has given a written consent for the publication of the paper.

\section{References}

1. NIELSEN H. Epidemiology of Schonlein-Henoch purpura. Acta Paediatr. Scand., 1988, 77 : 125-31.

2. SAULSBURY F. Clinical update: Henoch Schonlein purpura. Lancet, 2007, 369 : 976-8.

3. AGHA F, NOSTRANT T, KEREN D. Leucocytoclastic vasculitis (hypersensitivity angiitis) of the small bowel presenting with severe gastrointestinal hemorrhage. Am. J. Gastroenterol., 1986, 81 : 195-8. 